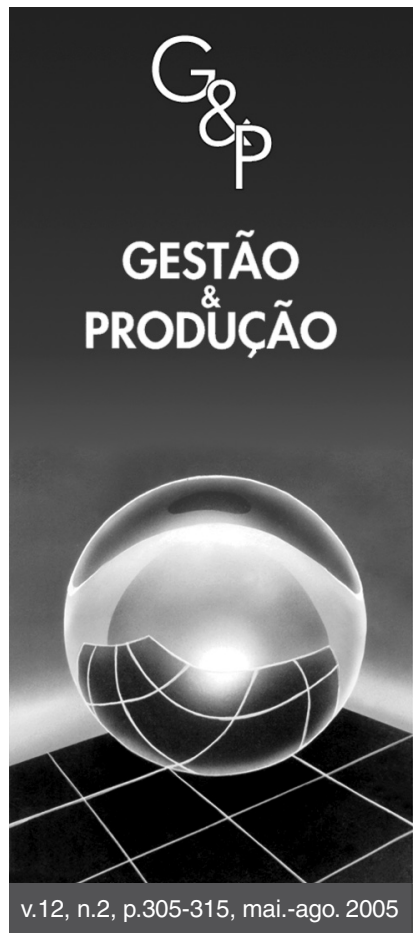

\title{
DEFINIÇÃO DE ATRIBUTOS DESEJÁVEIS PARA AUXILIAR A AUTO-AVALIAÇÃO DOS NOVOS SISTEMAS DE MEDIÇÃO DE DESEMPENHO ORGANIZACIONAL
}

\author{
Moacyr Amaral Domingues Figueiredo \\ Núcleo de Competitividade, Estratégia e Organização - LabCEO, \\ Departamento de Engenharia de Produção, UFF, \\ Rua Passo da Pátria, 156, CEP 24210-240, São Domingos, Niterói, RJ, \\ e-mail: lcfmadf@uol.com.br
}

Teresia Diana Lewe van Aduard de Macedo-Soares

Dep. de Administração e Escola de Negócio da Pontifícia Universidade Católica, Rua Marques de São Vicente, 225, CEP 22453-900, Rio de Janeiro, RJ, e-mail: pesquisa@strategy-research.com

Saul Fuks

Programa de Engenharia de Produção da COPPE, Centro de Tecnologia, UFRJ, Bloco F, CEP 21945-970, Cidade Universitária, Rio de Janeiro, RJ, e-mail: saul@ pep.ufrj.br

Lívia Cavalcanti Figueiredo

Departamento de Administração da Fundação Educacional Serra dos Órgãos, Av. Alberto Torres, 111, CEP 25964-005, Alto, Teresópolis, RJ, e-mail: Icfmadf@uol.com.br

Recebido em 30/10/2003 Aceito em 22/6/2005

\section{Resumo}

Este trabalho tem como principal objetivo definir Atributos Desejáveis (ADs) para orientar a auto-avaliação dos novos Sistemas de Medição de Desempenho Organizacional (SMDOs). Primeiramente, são explicados os objetivos da pesquisa que levou à definição do conjunto de ADs, bem como a relevância deste, e são apresentados o referencial teórico e a metodologia utilizada. Em seguida, é descrito como os ADs foram definidos a partir das diversas características de SMDO identificadas na literatura sobre o tema. Por fim, são apresentadas algumas conclusões e sugestões para pesquisas futuras.

Palavras-chave: sistemas de medição de desempenho, auto-avaliação, atributos desejáveis.

\section{Introdução}

A evolução dos Sistemas de Medição de Desempenho Organizacional (SMDOs) pode ser dividida em três fases distintas. A primeira, que foi do século XIV ao século XIX, quando surgiram as primeiras práticas de contabilidade para controle do processo produtivo e quando foram desenvolvidos os primeiros sistemas de administração e controle da produção. A segunda, que foi do início até os meados da década de 1980, quando a medição de desempenho passou formalmente a fazer parte do ciclo de planejamento e controle das organizações e a Pirâmide Du Pont foi o principal modelo de SMDO utilizado (Ghalayini e Noble, 1996). A terceira iniciou-se durante a década de 1980, estendendo-se até os dias de hoje, quando, com base nas diversas críticas feitas aos modelos de SMDOs tradicionais, novos modelos com múltiplas dimensões de desempenho foram propostos e características necessárias à sua eficácia foram identificadas na literatura sobre o tema.

Atualmente, as organizações têm demonstrado interesse pelos novos modelos de SMDOs, entretanto, a grande maioria tem encontrado dificuldades para implementálos. Cerca de 70\% (setenta por cento) dos novos SMDOs implementados não funcionaram adequadamente (Neely e Bourne, 2000).

Por outro lado, pesquisadores e praticantes reconhecem a auto-avaliação organizacional como uma das mais 
importantes práticas para disseminação e internalização de processos de mudança (Hillman, 1994; EFQM, 1995; Wiele et al., 1995; Wiele et al., 1996; Zink e Schmidt, 1998; Caffyn, 1999; Wiele et al., 2000). Apesar de tal importância, a auto-avaliação dos SMDOs tem sido relegada a um segundo plano. Até o momento, poucos estudos foram realizados para auto-avaliar os novos SMDOs. Constatou-se que a falta de procedimentos para auto-avaliação é uma das lacunas existentes no corpo de conhecimento sobre SMDOs (Figueiredo, 2003).

Este artigo objetiva contribuir à lacuna mencionada ao desenvolver, a partir de características identificadas na literatura sobre o tema, um conjunto de Atributos Desejáveis (ADs) para a auto-avaliação de SMDOs.

$\mathrm{O}$ artigo é dividido em quatro seções, além desta introdução. Na seção 2 são explicados os objetivos da pesquisa que levaram ao desenvolvimento do conjunto de ADs, bem como a relevância deste. Na seção 3 são apresentados os conceitos centrais ao referencial teórico. $\mathrm{Na}$ seção 4 é explicada a metodologia utilizada para a definição dos ADs. Na seção 5 são apresentadas as definições dos ADs e suas variáveis observáveis. Na última seção são apresentadas algumas conclusões e sugestões para pesquisas futuras.

\section{A pesquisa maior e sua relevância}

O desenvolvimento dos ADs em questão integra uma pesquisa mais abrangente, chamada aqui de pesquisa maior, cujo objetivo é responder à seguinte pergunta: "No caso de empresas que adotaram os novos sistemas de medição de desempenho, utilizados pelas organizações líderes no Brasil, como realizar auto-avaliações que possam contribuir para uma maior coerência entre o sistema de medição e a nova estratégia organizacional; aumentar a satisfação dos usuários do SMDO; aumentar a aceitação de uma nova cultura de medição; e contribuir para o aperfeiçoamento do sistema em questão?"' (Figueiredo, 2003).

Para responder à questão acima, foi desenvolvido um modelo para auxiliar a auto-avaliação de SMDO que tem, como variáveis independentes, os Atributos Desejáveis e, como variáveis dependentes, os resultados esperados da auto-avaliação do SMDO: a satisfação dos usuários do SMDO; a coerência do SMDO com a estratégia adotada pela organização; a aceitação de uma nova cultura de medição; e o aperfeiçoamento do SMDO (Figueiredo, 2003).

Esta pesquisa é relevante, visto que, até o momento, poucos esforços foram realizados para avaliar os novos SMDOs. O esforço em propor soluções para o problema da inadequação dos SMDOs tradicionais tem sido mais na proposição de novos modelos e metodologias de implementação do que na avaliação de tais sistemas.

\section{Definição dos conceitos centrais}

Os conceitos centrais da pesquisa foram desenvolvidos a partir dos resultados da revisão da literatura sobre as seguintes questões: 1) a evolução dos sistemas de medição de desempenho; 2) as principais críticas feitas aos sistemas tradicionais; 3 ) as propostas de novos modelos; 4) as características identificadas no corpo de conhecimento; e 5) os fundamentos da auto-avaliação organizacional.

Em seguida, são apresentadas as definições dos principais conceitos, tais como: Sistema de Medição de Desempenho Organizacional, Atributo Desejável e Ciclo de Auto-avaliação de SMDO.

O Sistema de Medição de Desempenho Organizacional - SMDO é definido nesta pesquisa como:

" $O$ conjunto de pessoas, processos, métodos, ferramentas e indicadores estruturados para coletar, descrever e representar dados com a finalidade de gerar informações sobre múltiplas dimensões de desempenho, para seus usuários dos diferentes níveis hierárquicos. Com base nas informações geradas, os usuários podem avaliar o desempenho de equipes, atividades, processos e da própria organização, para tomar decisões e executar ações para a melhoria do desempenho." (inspirado em Clark, 1995; Bititci et al., 2000; Neely et al., 2002).

Com relação ao conceito Atributo Desejável (AD) de um SMDO, na pesquisa, foi definido como:

"A característica geral de um SMDO que o qualifica e é estabelecida como base para sua avaliação." (inspirado em Clark, 1995)

O Ciclo de Auto-avaliação de SMDO é definido como:

"O ciclo sistemático e regular de análise crítica de um SMDO em relação a um modelo de avaliação baseado no conjunto de Atributos Desejáveis de SMDO. É realizado por pessoas pertencentes à organização, usuárias do SMDO. Tem como finalidades identificar as forças e fraquezas do SMDO, e propor ações planejadas para sua melhoria." (inspirado em EFQM 1993; Hilman 1994; Zink e Schmidt, 1998; Harrison e Shiron, 1999).

\section{Metodologia}

Para garantir que as melhores práticas fossem utilizadas para a definição dos ADs, artigos sobre o desenvolvimento e teste de teoria foram revisados, tais como: Kerlinger (1991), Lakatos e Marconi (1991), Sekaran (1992), Ahire et al. (1996), Handfield e Melnyk (1998), Melnyk e Handfield (1998), Meredith (1998), Wacker (1998), Amudson (1998), Malhotra e Grover (1998) e Boyer e Pagell (2000).

Segundo Kerlinger (1991) a teoria é composta por "um conjunto de constructos (conceitos) inter-relacionados, definições e proposições que apresentam uma visão 
sistemática do fenômeno, especificando relações entre variáveis, com o objetivo de explicar e predizer o fenômeno" (Kerlinger, 1991). Abrange dois domínios, o domínio teórico e o domínio operacional. Constructos ou conceitos são abstrações no domínio teórico que expressam características similares (Malhotra e Grover, 1998).

Os conceitos e os constructos têm significados semelhantes, mas apresentam uma importante distinção: enquanto o conceito expressa uma abstração, formada mediante a generalização de observações particulares, o constructo não pode ser tão facilmente ligado aos fenômenos que representa, pois é um conceito consciente e deliberadamente desenvolvido ou adotado com um propósito científico (Lakatos e Marconi, 1991).

A teoria tenta explicar fenômenos observados, fixando sistematicamente inter-relações entre constructos. Todavia, como os constructos são abstratos, os pesquisadores devem prover uma definição operacional daquilo que é observável. Esta definição operacional representa um símbolo ou uma variável cujos valores numéricos podem ser assinalados (Malhotra e Grover, 1998). Inspirado em Hayes (1998), Malhotra e Grover (1998) e Wacker (1998), adotou-se uma metodologia em duas etapas para definição dos ADs.

Na 1a. Etapa, após a revisão da literatura sobre características de SMDO, foram definidos os elementos que compõem a auto-avaliação de SMDO.

Na 2a. Etapa, os Atributos Desejáveis foram definidos e apresentadas suas respectivas variáveis observáveis.

\section{Definição dos atributos desejáveis}

\subsection{1a. Etapa - Definição dos elementos da auto-avaliação de SMDO}

De acordo com Hillman (1994), a auto-avaliação organizacional é composta por três elementos principais: 1) o modelo no sentido de referencial que define o arcabouço em função do qual o progresso da organização é avaliado; 2) a medição que é o meio utilizado para coletar dados sobre o progresso da organização em relação ao modelo utilizado; e 3) o gerenciamento que consiste nas atividades para administrar todo o processo de auto-avaliação.

Utilizando os elementos propostos por Hillman (1994), neste estudo, a auto-avaliação de SMDO teve a seguinte composição: 1) o conjunto de Atributos Desejáveis que representa o arcabouço teórico em função do qual o SMDO é avaliado; 2) o instrumento de auto-avaliação pelo qual as evidências e dados são coletados e que é composto pelas variáveis observáveis relacionadas a cada um dos ADs; e 3) o gerenciamento que compreende as atividades de administração do Ciclo de Auto-avaliação de SMDO.

Este estudo focalizou apenas os dois primeiros elementos da auto-avaliação: o modelo (o conjunto de ADs) e a medição (o instrumento de medição com suas variáveis observáveis). O terceiro elemento - as atividades de gerenciamento da auto-avaliação - não faz parte do escopo deste trabalho.

\subsection{2a. Etapa - Definição dos atributos de- sejáveis e suas variáveis observáveis}

Inicialmente, foirealizadaumaextensarevisãodaliteratura sobre o tema, visando identificar características necessárias à eficácia dos SMDOs. Os principais autores consultados foram: Lynch e Cross (1991); Hronec (1994); Bititci (1995a); Bititci (1995b); Clark (1995); Neely et al. (1995); Ghalayini e Noble (1996); Neely et al. (1996); Bititci et al. (1997); Ghalayini et al. (1997); Neely et al. (1997); Martins (1998); Bititci et al. (2000); Bourne et al. (2000); Neely etal. (2000); De Toni e Tonchia (2001); Neely et al. (2001); Kennerly e Neely (2002); Neely et al. (2002). Cento e vinte oito características foram identificadas na literatura e podem ser consultadas em Figueiredo (2003).

A partir da reflexão dos resultados da revisão da literatura, foram definidos 9 (nove) Atributos Desejáveis: Aprendizado, Análise Crítica, Balanceamento, Clareza, Dinamismo, Integração, Alinhamento, Participação e Relacionamento Causal. A seguir são apresentadas as definições desses ADs e suas respectivas variáveis observáveis.

\subsection{Aprendizado organizacional}

A discussão sobre as organizações voltadas para o aprendizado (learning organizations) tem ocupado um espaço significativo na literatura gerencial desde a década de 1990, sendo que a sua popularização deve-se, em boa medida, ao livro "A Quinta Disciplina” de Senge (1996).

Segundo Garvin (1993) "uma organização voltada para o aprendizado é uma organização hábil na criação, aquisição e transferência de conhecimentos, e na modificação do seu comportamento para refletir os novos conhecimentos e percepções". Neste tipo de organização, os novos conhecimentos são absorvidos e transmitidos aos seus membros de forma a contribuir para a melhoria contínua dos processos da organização e o aumento de sua competitividade.

Segundo Kaplan e Norton (1996b), um dos principais objetivos do SMDO é fornecer informações que facilitem o processo de aprendizado organizacional. Com as informações fornecidas pelo SMDO, os tomadores de decisão podem questionar pressupostos e avaliar se as teorias com que estão trabalhando continuam coerentes com as evidências, as observações e as experiências reais (Kaplan e Norton, 1996b).

O SMDO deve fornecer informações que possibilitem aos tomadores de decisão perceberem não só se a estratégia está sendo executada de acordo com o planejado, mas, também, se a estratégia planejada continua sendo viável e bem sucedida. 
Para avaliar se o SMDO está facilitando o processo de aprendizagem organizacional o seguinte Atributo Desejável foi definido:

"Aprendizado Organizacional é a capacidade do SMDO fornecer informações que facilitem o questionamento, a avaliação ou a inovação do SO, de forma a contribuir não só para a criação, aquisição e transferência de conhecimentos, mas também para modificação do comportamento do SO, refletindo os novos conhecimentos e percepções obtidos." (Garvin, 1993; FPNQ, 2002; Kaplan e Norton, 1996b)

Baseado na definição de Aprendizado Organizacional e nas características identificadas na literatura, foi proposto um conjunto de 7 (sete) variáveis observáveis para mensurar esse Atributo Desejável que é apresentado no Anexo.

\subsection{Análise crítica}

Para que a organização possa melhorar continuamente seu desempenho, deve ter um SMDO que forneça informações que possibilitem a avaliação do seu desempenho global. O SMDO que preenche tal requisito coleta informações sobre o ambiente externo e interno da organização, utiliza informações comparativas que permitem avaliar o desempenho dos processos em relação a padrões externos e internos, utiliza mecanismos que fornecem relações de causa e efeito entre os indicadores e fornece informações quantitativas e qualitativas relevantes.

As informações fornecidas pelo SMDO permitem que seus usuários possam compará-las com os objetivos e as prioridades da organização, levantem problemas e proponham soluções para a melhoria do desempenho.

Para avaliar o quanto o SMDO ajuda na avaliação do desempenho da organização, o Atributo Desejável Análise Crítica é definido como: "a capacidade do SMDO fornecer informações que possibilitem uma verificação profunda e global do desempenho organizacional com a finalidade de identificar problemas e propor soluções" (adaptado de Ghalayini et al., 1997; Bititci et al., 2000; Nelly et al., 2002; FPNQ, 2002).

Baseado na definição do Atributo Desejável Análise Crítica e nas características identificadas na literatura, é proposto um grupo de 7 (sete) variáveis observáveis para avaliar o SMDO em relação ao Atributo Desejável definido e que são apresentadas no Anexo.

\subsection{Balanceamento}

No passado, os SMDOs utilizados pela maioria das organizações eram tradicionalmente compostos por indicadores financeiros, relacionados apenas aos interesses dos acionistas. Esses indicadores forneciam somente informações de curto prazo e não indicavam como as organizações poderiam melhorar seu desempenho no futuro (Kaplan e Norton, 1996b).
Atualmente, os SMDOs devem medir não somente o desempenho financeiro que está relacionado aos interesses dos acionistas, mas também a satisfação dos clientes, a qualidade dos produtos e serviços, a satisfação do funcionário e o clima organizacional, a imagem que a sociedade tem da organização, o impacto ambiental de seus produtos, o desempenho dos seus fornecedores, a inovação e o aprendizado organizacional, e a eficácia e eficiência dos processos de negócio (Figueiredo, 2003).

Dentro desse contexto, o Atributo Desejável Balanceamento é definido como "a capacidade do SMDO de fornecer informações, segundo diferentes dimensões de desempenho, que possibilitem obter uma percepção multidimensional do comportamento da organização". (Kaplan e Norton, 1996a; Neely et al., 2002; FPNQ, 2002).

Baseado na definição do Atributo Desejável Balanceamento e nas características identificadas na literatura, é proposto um conjunto de 5 (cinco) variáveis observáveis para mensurar este $\mathrm{AD}$ e que é apresentado no Anexo.

\subsection{Clareza}

Os indicadores utilizados no SMDO devem ser claramente definidos e apresentados de forma a fornecerem informações e não apenas dados. Essas características ajudam o usuário do SMDO a ter uma percepção clara do desempenho organizacional.

Quanto à sua definição, é importante que o objetivo, a fórmula de obtenção do índice, as fontes de dados, os referenciais comparativos e metas estejam claramente definidos.

Quanto à apresentação do indicador, é importante que seus índices sejam apresentados em gráficos, confeccionados de forma que o usuário perceba facilmente como o produto, processo ou sistema está se comportando ao longo do tempo.

Dentro deste contexto o Atributo Desejável Clareza é definido como "a capacidade do SMDO utilizar indicadores de fácil entendimento para os usuários dos diferentes níveis hierárquicos da organização". (Sink e Smith, 1993; Sink e Tuttlie, 1993; Neely et al., 2000).

Baseado na definição do Atributo Desejável Clareza e nas características identificadas na literatura, é proposto um conjunto de 10 (dez) variáveis observáveis para mensurar este $\mathrm{AD}$ e que é apresentado no Anexo.

\subsection{Dinamismo}

A maioria das organizações utiliza SMDOs estáticos, isto é, sistemas que não possuem mecanismos que permitam sua adaptação rápida às mudanças ocorridas no ambiente interno e externo da organização. Esta falta de adaptabilidade às mudanças tem um efeito negativo sobre a agilidade e a capacidade de resposta da organização.

Para que o SMDO seja dinâmico, ele deve ser sensível às mudanças ocorridas nos ambientes interno e externo 
da organização e deve fornecer informações sobre tais mudanças de forma a permitir que a organização faça uma revisão dos seus objetivos internos e desdobre as mudanças feitas para as partes críticas da organização, assegurando o alinhamento contínuo e a manutenção dos ganhos alcançados pelos programas de melhoria organizacional (Bititci et al., 2000).

Dentro deste contexto, o Atributo Desejável Dinamismo é definido como "a capacidade do SMDO de monitorar continuamente o ambiente externo e interno da organização de forma a fornecer informações pertinentes no momento em que são solicitadas, adaptarse rapidamente às mudanças ocorridas no ambiente interno e externo da organização e permitir o acompanhamento do desempenho da organização ao longo do tempo". (Ghalayini et al., 1997; Bititci et al., 1998; Bititci et al., 2000).

Para facilitar a sua mensuração, o Atributo Desejável Dinamismo foi desdobrado em três subatributos que medem seus aspectos específicos: Agilidade, Flexibilidade e Monitoramento.

O primeiro subatributo do Dinamismo está relacionado à rapidez com que as informações pertinentes são disponibilizadas. Para isso, o subatributo Agilidade é definido como "a capacidade do SMDO disponibilizar as informações pertinentes no momento em que são solicitadas pelos usuários dos diferentes níveis hierárquicos da organização".

O segundo subatributo focaliza o aspecto do Dinamismo relacionado à rapidez com que o SMDO se adapta às mudanças ocorridas. Para isso, o subatributo Flexibilidade é definido como "a capacidade do SMDO adaptarse rapidamente às mudanças no ambiente interno e externo da organização e às necessidades dos usuários dos diferentes níveis hierárquicos".

O terceiro subatributo focaliza o aspecto do Dinamismo relacionado à detecção de problemas potenciais. Para isso o subatributo Monitoramento é definido como "a capacidade do SMDO acompanhar continuamente o ambiente externo e interno da organização a fim de detectar as ocorrências de problemas potenciais".

Baseado na definição do Atributo Desejável Dinamismo e seus subatributos, bem como nas características identificadas na literatura, são propostas 13 (treze) variáveis observáveis e que são apresentadas no Anexo.

\subsection{Integração}

O SMDO é um sistema de informação que faz parte do processo de gestão do desempenho global. Para isso, trata-se de apoiar o processo de desdobramento dos objetivos estratégicos e táticos do negócio, de assimilar as informações relevantes dos principais sistemas de informação da organização, e de agregá-las de forma a fornecer as informações-chave para o processo decisório da organização.
Nesse contexto, o Atributo Desejável Integração é definido como "a capacidade do SMDO interagir com todas as partes da organização e seus principais sistemas de informação". (Ghalayini et al., 1997; Bititci et al., 1998; Bititci et al., 2000).

Baseado na definição do Atributo Desejável Integração e nas características identificadas na literatura, são propostas 4 (quatro) variáveis observáveis para representar esse constructo e que são apresentadas no Anexo.

\subsection{Alinhamento}

A gestão do desempenho organizacional é o processo pelo qual a organização administra seu desempenho, alinhado com as estratégias e os objetivos corporativos e funcionais. Este processo tem como objetivo fornecer um sistema de controle com realimentação, em que as estratégias corporativas e funcionais são desdobradas para todos os processos de negócio, atividades, tarefas e pessoal, e a realimentação é obtida por meio do SMDO, para apoiar as decisões apropriadas de gestão.

Nesta linha de pensamento, o Atributo Desejável Alinhamento é definido como "a capacidade do SMDO utilizar indicadores vinculados com a estratégia e os principais processos organizacionais, combinados, interligados ou agrupados de forma a possibilitar uma percepção global do desempenho organizacional". (Kaplan e Norton, 1996b; Ghalayini et al., 1997; Bititci et al., 1998, 2000; Neely et al. 2000).

Baseado na definição do Atributo Desejável Alinhamento e nas características identificadas na literatura, são propostas 7 (sete) variáveis observáveis para representar esse constructo e que são apresentadas no Anexo.

\subsection{Participação}

A medição não pode ser de responsabilidade apenas da direção, deve ser dividida com os gerentes, supervisores e todo o pessoal do "chão de fábrica". Para isso, representantes dos usuários dos diferentes níveis hierárquicos devem participar ativamente do desenvolvimento, implementação, avaliação e aperfeiçoamento do SMDO.

Nesse contexto, o Atributo Desejável Participação é definido como "a capacidade do SMDO permitir que representantes dos diferentes grupos de usuários e interessados (fornecedores, controladores, acionistas, comunidade,etc.) tomem parte ativa em todas as fases do seu ciclo de vida (desenvolvimento, implementação, utilização, avaliação e aperfeiçoamento)". (Dixon et al., 1990; Thor, 1993 Ghalayini et al., 1997).

Baseado na definição do Atributo Desejável Participação e nas características identificadas na literatura, são propostas 5 (cinco) variáveis observáveis e que são apresentadas no Anexo.

\subsection{Relacionamento causal}

O relacionamento causal explica como a organização 
pretende alcançar seus objetivos estratégicos. Com ele, é possível identificar quais os resultados esperados e os possíveis pontos de alavancagem da organização (Neely e Bourne, 2000).

O SMDO deve tornar explícitas as relações causais entre os indicadores para que elas possam ser gerenciadas e validadas (Kaplan e Norton, 1996b). Tais relações não só ajudam os usuários do SMDO a perceberem como as ações executadas estão impactando os indicadores de desempenho, bem como a entenderem as conseqüências das decisões tomadas (Kaplan e Norton, 2001).

Nesse contexto, o Atributo Desejável Relacionamento Causal é definido como "a capacidade do SMDO interrelacionar os diversos indicadores de forma a facilitar o entendimento das relações de causa e efeito entre a estratégia, processos e indicadores da organização". (Kaplan e Norton, 1996b; Neely et al., 2002).

Baseado na definição do Atributo Desejável Relacionamento Causal e nas características identificadas na literatura, são propostas 5 (cinco) variáveis observáveis e que são apresentadas no Anexo.

\section{Conclusões}

Este trabalho partiu da constatação de que, apesar da grande evolução dos SMDOs e das técnicas de auto-avalia- ção organizacional nas últimas duas décadas do século XX, pouca atenção foi dada à auto-avaliação dos SMDOs.

A partir da revisão da literatura sobre os novos modelos de SMDOs, foram definidos 9 (nove) Atributos Desejáveis e 63 (sessenta e três) variáveis observáveis para auxiliar a auto-avaliação de SMDO. Os ADs definidos foram: Aprendizado Organizacional, Análise Crítica, Balanceamento, Clareza, Dinamismo, Integração, Alinhamento, Relacionamento Causal e Participação. O AD Dinamismo foi decomposto em 3 (três) subatributos: Agilidade, Flexibilidade e Monitoramento.

A definição do conjunto de Atributos Desejáveis é a principal contribuição deste trabalho, uma vez que apesar da existência de diversas características na literatura sobre medição de desempenho organizacional, elas ainda não haviam sido estudadas com o intuito de definir um conjunto de características-chave para a avaliação de SMDO.

A principal limitação deste trabalho diz respeito ao fato de que os novos modelos de SMDO podem possuir outras características que não foram incluídas nesse estudo.

Como direcionamento para futuras pesquisas relativas ao presente tema, sugere-se a validação estatística do conjunto de ADs em organizações comprovadamente reconhecidas pelas práticas de excelência em auto-avaliação e medição do desempenho organizacional. 


\section{Anexo}

\section{Atributos desejáveis e suas variáveis observáveis}

$\begin{array}{lll}\text { Atributos } & \text { VO } & \text { Variáveis Observáveis (VO) } \\ \text { Desejáveis } & \end{array}$

Aprendizagem

Organizacional

APRE1

APRE2

APRE3

APRE4

APRE5

APRE6

APRE7

Análise

Crítica

\section{O Sistema de Medição de Desempenho Organizacional da minha empresa...}

... fornece informações que auxiliam nas iniciativas de melhoria de desempenho da empresa.

... fornece informações que ajudam a entender melhor os principais processos da empresa.

... fornece informações que ajudam a perceber o comportamento da empresa ao longo do tempo.

... fornece informações que facilitam a identificação de ações corretivas para melhoria do desempenho da empresa.

fornece informações que servem de base para debate entre os usuários dos diversos níveis hierárquicos da empresa.

\section{O Sistema de Medição de Desempenho Organizacional da minha empresa...}

AVAL1 ... fornece informações que proporcionam uma visão do desempenho passado, presente e futuro da empresa.

AVAL2 $\quad \ldots$ as informações comparativas facilitam a análise crítica do desempenho global da empresa.

AVAL3 ... as informações qualitativas facilitam a análise crítica do desempenho global da empresa.

AVAL4 ... as informações sobre o ambiente externo facilitam a análise crítica do desempenho global da empresa.

AVAL5 ... as informações sobre as relações de causa e efeito entre os indicadores facilitam a análise crítica do desempenho global da empresa.

AVAL6 ... utiliza indicadores que são classificados, integrados e correlacionados para apoiar a análise crítica do desempenho global.

AVAL7 ... utiliza indicadores com metas de curto e de longo prazo que facilitam a análise crítica do desempenho global da empresa.

Balanceamento O Sistema de Medição de Desempenho Organizacional da minha empresa...

BAL1 ... utiliza múltiplas dimensões de desempenho (por exemplo: financeira, cliente, processos, inovação, etc.).

BAL2 ... utiliza um conjunto de indicadores que ajuda a ter uma visão multidimensional do desempenho organizacional.

BAL3 ... fornece informações que facilitam avaliar se as expectativas e necessidades dos principais interessados estão sendo satisfeitas.

BAL4 ... fornece informações que facilitam a identificação de oportunidades e ameaças do ambiente externo da empresa.

BAL5 ... fornece informações que facilitam a identificação de pontos fortes e oportunidades de melhoria do ambiente interno da empresa.

Clareza O Sistema de Medição de Desempenho Organizacional da minha empresa...

CLA1 ... as definições dos indicadores são entendidas por todos os usuários.

CLA2 ... os índices são calculados por meio de fórmulas matemáticas claramente definidas.

CLA3 ... os objetivos dos indicadores estão claramente definidos.

CLA4 ... para cada indicador estão definidos quais os usuários que utilizam suas informações para a tomada de decisão.

CLA5 ... os indicadores possuem referenciais com os quais podem ser comparados.

CLA6 ... os indicadores têm metas definidas claramente.

CLA7 ... são definidos claramente quais os dados que devem ser coletados para obtenção dos índices.

CLA8 ... as fontes de dados estão claramente definidas.

CLA9 ... apresenta as informações de forma visível para todos os usuários.

CLA10 ... apresenta os dados, utilizando gráficos em lugar de tabelas. 


\begin{tabular}{|c|c|c|}
\hline $\begin{array}{l}\text { Atributos } \\
\text { Desejáveis }\end{array}$ & VO & Variáveis Observáveis (VO) \\
\hline \multirow[t]{5}{*}{ Agilidade } & & O Sistema de Medição de Desempenho Organizacional da minha empresa... \\
\hline & AGI1 & ... fornece informações no momento em que o usuário necessita. \\
\hline & AGI2 & ... utiliza indicadores relevantes para a tomada de decisão. \\
\hline & AGI3 & ... fornece as informações de que o usuário precisa para a tomada de decisões. \\
\hline & AGI4 & ... fornece "feedback" rápido e preciso. \\
\hline \multirow[t]{7}{*}{ Flexibilidade } & & O Sistema de Medição de Desempenho Organizacional da minha empresa... \\
\hline & FLEX1 & ... os indicadores podem ser facilmente modificados quando necessário. \\
\hline & FLEX2 & ... adapta-se às necessidades dos diferentes grupos de usuários. \\
\hline & FLEX3 & ... permite a modificação de metas e padrões de desempenho. \\
\hline & FLEX4 & ... é difícil de ser manipulado. \\
\hline & FLEX5 & ... fornece informações que auxiliam no acompanhamento do desempenho da empresa ao longo do tempo. \\
\hline & FLEX6 & ... utiliza indicadores de desempenho orientados para médio e longo prazo. \\
\hline \multirow[t]{4}{*}{ Monitoramento } & & O Sistema de Medição de Desempenho Organizacional da minha empresa... \\
\hline & MON1 & ... monitora continuamente o ambiente externo da empresa a fim de detectar mudanças ocorridas. \\
\hline & MON2 & ... monitora continuamente o ambiente interno da empresa a fim de detectar mudanças ocorridas. \\
\hline & MON3 & ... utiliza sinais de alarme para detectar problemas potenciais. \\
\hline \multirow[t]{5}{*}{ Integração } & & O Sistema de Medição de Desempenho Organizacional da minha empresa... \\
\hline & INT1 & ... interage com os principais sistemas de informação da empresa. \\
\hline & INT2 & ... está bem integrado com o planejamento e monitoramento da empresa. \\
\hline & INT3 & ... está bem estabelecido em todos os níveis da empresa. \\
\hline & INT4 & ... facilita a cooperação entre os departamentos. \\
\hline \multirow[t]{8}{*}{ Alinhamento } & & O Sistema de Medição de Desempenho Organizacional da minha empresa... \\
\hline & ALI1 & ... permite combinar e/ou agregar os diversos indicadores. \\
\hline & ALI2 & ... ajuda a perceber como a estratégia da empresa esta sendo executada. \\
\hline & ALI3 & ... utiliza indicadores relacionados com as principais metas da empresa. \\
\hline & ALI4 & $\begin{array}{l}\text {... utiliza indicadores que medem o desempenho de todas as etapas dos principais processos de negócio da } \\
\text { empresa. }\end{array}$ \\
\hline & ALI5 & ... utiliza indicadores que relacionam os principais processos com a estratégia da empresa. \\
\hline & ALI6 & ... utiliza indicadores que ajudam a integrar os principais processos de negócio da empresa. \\
\hline & ALI7 & ... agrega os indicadores, segundo a estrutura hierárquica da empresa. \\
\hline
\end{tabular}

\begin{tabular}{|c|c|c|}
\hline $\begin{array}{l}\text { Atributos } \\
\text { Desejáveis }\end{array}$ & VO & Variáveis Observáveis (VO) \\
\hline \multirow[t]{6}{*}{ Participação } & & O Sistema de Medição de Desempenho Organizacional da minha empresa... \\
\hline & PART1 & ... foi desenvolvido com a participação ativa de representantes dos seus diferentes grupos de usuários. \\
\hline & PART2 & ... foi desenvolvido levando em conta as necessidades de informação dos diferentes grupos de usuários. \\
\hline & PART3 & ... foi implementado com a participação ativa de representantes dos diferentes grupos de usuários. \\
\hline & PART4 & ... pode ser modificado em função de sugestões e reclamações dos diferentes grupos de usuários. \\
\hline & PART5 & ... é avaliado e melhorado com a participação ativa dos representantes dos diferentes grupos de usuários. \\
\hline \multirow{6}{*}{$\begin{array}{l}\text { Relacionamento } \\
\text { Causal }\end{array}$} & & O Sistema de Medição de Desempenho Organizacional da minha empresa... \\
\hline & $\mathrm{RC} 1$ & ... fornece informações que facilitam a identificação de pontos críticos e prioridades para a empresa. \\
\hline & $\mathrm{RC} 2$ & $\begin{array}{l}\text {... fornece informações que facilitam o entendimento das relações de causa e efeito entre as ações executa- } \\
\text { das e os indicadores de desempenho. }\end{array}$ \\
\hline & $\mathrm{RC} 3$ & ... fornece informações que facilitam a identificação das relações de causa e efeito entre os indicadores. \\
\hline & $\mathrm{RC} 4$ & $\begin{array}{l}\text {... fornece informações que facilitam o relacionamento entre o desempenho passado e o planejamento do } \\
\text { desempenho futuro da empresa. }\end{array}$ \\
\hline & $\mathrm{RC} 5$ & ... fornece informações que ajudam a perceber as consequiências financeiras das decisões tomadas. \\
\hline
\end{tabular}


AHIRE, S. L.; GOLHAR, D. Y.; WALLER, M. A. Development and validation of TQM implementation constructs. Decision Science, v. 27, n. 1, p. 23-70, 1996.

AMUDSON, S. D. Relationships between theory-driven empirical research in operations management and other disciplines. Journal of Operations Management, v. 16, n. 4, p. 341-359, 1998.

BITITCI, U. S. Measuring the integrity of your business. Management Decisions, v. 33, n. 7, p. 10-18, 1995a.

Modeling of performance measurement systems in manufacturing enterprises. International Journal of Production Economics, v. 42, n. 2, p., 137-147, 1995 b.

BITITCI, U. S.; TURNER, T.; BEGEMANN, C. Dynamics of performance measurement systems. International Journal of Operations \& Production Management, v. 20, n. 6, p. 692-704, 2000.

BITITCI, U. S.; CARRIE, A. S.; McDEVITT, L. Integrated performance measurement systems: a development guide. International Journal of Operations \& Production Management, v. 17, n. 5, p. 692-704, 1997.

BITITCI, U. S.; CARRIE, A.; TURNER, T. Diagnosing the integrity of your performance measurement system. Control, April, p. 9-13, 1998.

BOURNE, M.; MILLS, J.; WILCOX, M.; NEELY, A.; PLATTS, K. Designing, implementing and updating performance measurement systems. International Journal of Operations \& Production Management, v. 20, n.7, p. 754-771, 2000.

BOYER, K. K.; PAGELL, M. Measurement issues in empirical research: improving measures of operations strategy and advanced manufacturing technology. Journal of Operations Management, v. 18, n. 3, p. 361-374, 2000.

CAFFYN, S. Development of a continuous improvement self-assessment tool, International Journal of Operations \& Production Management, v. 19, n. 11, p. 1138-1153, 1999.

CLARK, L. A. Development, application, and evaluation of an organizational performance measurement system. 1995. Tese (Doutorado em Industrial Engineering) - Industrial \& Systems Engineering Departament, Virginia Polytech Institute and State University, Blacksburg, 1995.

DE TONI, A.; TONCHIA, S. Performance measurements systems, models, characteristics and measures. International Journal of Operations \& Production Management, v. 21, n. 1-2, p. 48-70, 2001.
DIXON, J. R. ; NANNI, A., J. E; VOLLMANN, T. J. The New Performance Challenge: Measuring Operations for World-Class Competition. Homewood, IL: Irwin Professional Publishing: Dow Jones-Irwin, 1990.

EUROPEAN FOUDATION FOR QUALITY MANAGEMENT. European Foundation for Quality Management: self-assessment based on the European Model for Total Quality, The European Foundation for Total Quality, Brussels, 1995.

. European Foundation for Quality Management: self-assessment based on the European Model for Total Quality. Brussels, 1993.

FIGUEIREDO, M. A. D. Sistemas de Medição de Desempenho Organizacional: um modelo para auxiliar a sua auto-avaliação. 2003. 274 f.. Tese (Doutorado em Engenharia de Produção) - Coordenação dos Programas de Pós-graduação em Engenharia, Universidade Federal do Rio de Janeiro, Rio de Janeiro, 2003.

FUNDAÇÃO PARA O PRÊMIO NACIONAL DA QUALIDADE. Critérios de Excelência, São Paulo - SP, 2002.

GARVIN, D. A. Manufacturing strategic planning. California Management Review. Summer, p. 85-105, 1993.

GHALAYINI, A. M.; NOBLE, J. S. The changing basis of performance measurement. International Journal of Operations \& Production Management, v. 16, n. 8, p. 63-80, 1996.

GHALAYINI, A. M.; NOBLE, J. S.; CROWE, T. J. An integrate dynamic performance measurement system. International Journal of Production Economic, v. 48, n. 3, p. 207-225, 1997.

HANDFIELD, R. B.; MELNYK, S. A. The scientific theorybuilding process: a primer using the case of TQM. Journal of Operations Management, v. 16, n. 4, p. 321-339, 1998.

HARRISON, M., SHIROM, A. Organizational Diagnosis and Assessment, Thousand Oaks, California: Sage Publications, Inc., 1999.

HAYES, B. E. Measuring Customer Satisfaction: survey design, use, and statistical analysis methods. Milwaukee, Wisconsin: ASQC Quality Press, 1998.

HILLMAN, P. G. Making Self-assessment Successful. The TQM Magazine, v. 6, n. 3, p. 29-31, 1994.

HRONEC, S. M. Sinais Vitais: usando medidas de desempenho da qualidade, tempo e custos para traçar a rota para o futuro. Rio de Janeiro: Makron Books, 1994. 
KAPLAN, R. S.; NORTON, D. P. Organização orientada para a estratégia: como as empresas que adotam o balanced scorecard prosperam no novo ambiente de negócios, Rio de Janeiro: Campus, 2001.

A estratégia em ação: balanced scorecard, Boston, MA: Harvard Business School Press, 1996b.

. Using the balanced scorecard as a strategic management system. Harvard Business Review, v. 74, n. 1, p. 75-85, 1996a.

KENNERLEY, M. P.; NEELY, A. Performance measurement frameworks: a review. In: NEELY, A. Business Performance Measurement: Theory and Practice, Cambridge University Press, 2002.

KERLINGER, F. N. Foundations of behavioral research. New York: Holt, Rineahart \& Winston, 1991.

LAKATOS, E. M.; MARCONI, M. A. Metodologia Cien-

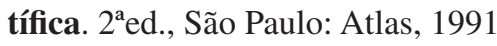

LYNCH, R. L.; CROSS-, K. F. Measure Up! Yardstick for Continuous Improvement. Cambridge, MA: Blackwell Business, 1991.

MALHOTRA, M. K., GROVER, V. An assessment of survey research in POM: from constructs to theory. Journal of Operations Management, v. 16, n. 4, p. 407-425, 1998.

MARTINS, R. A. Sistemas de medição de desempenho: um modelo para estruturação do uso. 1998. Tese (Doutorado em Engenharia de Produção) - Escola Politécnica da Universidade de São Paulo, Universidade de São Paulo, São Paulo, 1998.

MELNYK, S. A., HANDFIELD, R. B. May you live in interesting times ... the emergence of theory-driven empirical research. Journal of Operations Management, v. 16, n. 4, p. 311-319, 1998.

MEREDITH, J. Building operations management theory through case and field research. Journal of Operations Management, v. 16, n. 4, p. 441-454, 1998.

NEELY, A. D.; GREGORY, M. J.; PLATTS, K. W. Performance measurement system design - a literature review and research agenda. International Journal of Operations \& Production Management, v. 15, n. 4, p. 80-116, 1995.

NEELY, A. et al. Performance measurement system design: developing and testing a process-based approach. International Journal of Operations \& Production Management, v. 20, n. 10, p. 1119-1145, 2000.
NEELY, A. et al. Designing performance measures: a structured approach. International Journal of Operations \& Production Management, v. 19, n. 2, p. 205-228, 1997.

NEELY, A.; ADAMS, C.; CROWE, P. The performance prism in practice. Measuring Business Excellence, v. 5, n. 2, p. 6-13, 2001.

NEELY, A.; GREGORY, M. J.; PLATTS, K. W. Getting the Measure of your Business. Cambridge: Manufacturing Engineering Group, University of Cambridge, 1996.

NEELY, A.; BOURNE, M. Why measurement initiates fail. Measuring Business Excellence, v. 4, n. 4, 2000.

NEELY, A; ADAMS, C.; KENNERLEY, M. The Performance Prism: the scorecard for measuring and managing business success. London, Great Britain: Prentice Hall, Pearson Education Limited, 2002.

SEKARAN, U. Research methods for business: a skillbuilding approach. $2^{\mathrm{a}}$ ed., Singapore: John Wiley \& Sons, Ind., 1992.

SENGE, P. M. e al. A Quinta Disciplina - Arte , teoria e prática da organização de aprendizagem. São Paulo: Best Seller, 1996.

SINK, D. S.; SMITH, G. L. Performance linkages: understanding the role of planning, measurement, and evaluation in large-scale organizational change. Quality and Productivity Management, v. 10, n. 3, p. 27-36, 1993.

SINK, D. S; TUTTLE, T. C. Planejamento e Medição para a Performance, Rio de Janeiro: Qualitymark, 1993.

THOR, C. G. Ten rules for building a measurement system. Quality and Productivity Management, v. 9, n. 1, p. 7-10, 1993.

WACKER, J. G. A definition of theory: research guidelines for different theory-building research methods in operations management. Journal of Operations Management, v. 16, n. 4, p. 361-385, 1998.

WIELE, V. et al. Improvement in organizational performance and self-assessment practices by selected American firms. Quality Management Journal, v. 7, n. 2, p. 8-22, 2000.

WIELE, V. et al.. Self-assessment: A study of progress in Europe's leading organizations in quality management practices. International Journal of Quality \& Reliability Management, v. 13, n. 1, p. 84-104, 1996.

WIELE, V. et al.. State-of-the-art study on self-assessment. The TQM Magazine, v. 7, n. 4, p. 13-17, 1995.

ZINK, K. J.; SCHMIDT, A. Practice and implementation of self-assessment. International Journal of Quality Science, v. 3, n. 2, p. 147-170, 1998. 


\section{DEFINITION OF DESIRABLE ATTRIBUTES TO AID SELF-ASSESSMENT IN NEW ORGANIZATIONAL PERFORMANCE MEASUREMENT SYSTEMS}

\section{Abstract}

The main purpose of this paper is to define Desirable Attributes (DAs) to aid in the self-assessment of new organizational Performance Measurement Systems (PMSs). We begin by setting forth the objectives of the research that led to the definition of a set of DAs, as well as an explanation of their relevance, and a discussion of the theoretical basis and methods employed. An explanation is then given of how the DAs were defined based on the various characteristics identified within the scope of an extensive review of the literature on the subject. This paper closes with some conclusions and suggestions for future research.

Keywords: performance measurement systems, self-assessment, desirable attributes. 